\title{
Remarques sur l'évolution du réseau urbain en Suisse de 1850 à 1960
}

Jean-Luc Piveteau

Dans une publication antérieure ${ }^{1}$, nous avons étudié la distribution des communes de la Suisse en fonction de leur taille en 1850 et en 1960. Les résultats de cette confrontation nous ont permis d'apprécier les changements intervenus dans la texture du réseau des agglomérations - des plus petites aux plus grandes - entre ces deux dates-repères. Mais ils n'ont, toutefois, constitué qu'une approche, car ils laissaient ouvert le problème de l'identité des localités mutantes: de ce que certaines catégories statistiques se soient enrichies et d'autres se soient appauvries, il serait abusif de tirer en effet, que la compensation numérique s'est effectuée selon un principe de moindre modification. Rien n'autorise à dire que si, par rapport à celle de 1850 la pyramide de 1960 accuse une diminuation de nombre à la hauteur des très petits centres, et un accroissement inverse au niveau des villes, ce soit sous l'effet d'un simple transfert des communes manquantes du bas vers les communes désormais plus nombreuses du haut. Au surplus, à l'intérieur même des tranches de taille qui, quant aux effectifs de villages ou de cités ont peu changé - voire n'ont pas changé - des mouvements non négligeables de communes se déclassant et de communes progressant, ont pu avoir lieu qui n'apparaissent évidemment pas. Bref, s'il est légitime, au terme d'une semblable recherche, de conclure que, moyennant certaines déformations localisables quant aux strates qu'elles affectent, le réseau des impacts du peuplement en 1960 ressemble par sa texture générale au réseau de 1850 , il est exlu d'affirmer d'emblée que ce sont toujours les mêmes noms de lieux que l'on doit retrouver en tête, au centre ou en bas de cette hiérarchie fondée sur le rang. Seule une étude nominale des 3095 communes helvétiques permet de lever cette hypothèque. Nous l'avons effectuée en procédant à l'aide d'un tableau à double entrée. Sur chacun des axes nous avons porté les dix classes d'agglomérations arbitrairement mais coutumièrement distinguées l'un de ces axes se rapportant à 1850 , et l'autre à 1960. Ensuite de quoi, chaque localité a été inscrite à l'emplacement de celle des 100 positions théoriques possibles qui correspondit à son cas. Il en est résulté une disposition grossièrement linéaire, et relativement peu dispersée, dont nous voudrions rendre compte ici.

La première observation fondamentale est la suivante. Que l'on cherche l'origine - c'est à dire, dans l'optique de notre thème; la classe numérique des communes qui, en 1960, comptaient de 0 à 250 , de 250 à $500 \ldots$. . de 100000 à 700000 habitants; ou que l'on veuille, au contraire, connaître le destin, cent dix ans plus tard, des communes qui, en 1850 , rassemblaient de 0 à 250,250 à $500 \ldots 20000$ à 50000 habitants, notre matrice montre que la coïncidence diminue avec la taille. En d'autres termes, plus on regarde vers les rangs inférieurs, plus le nombre des communes ayant conservé en un siècle, leur catégorie de départ, augmente (cf. fig.). On est tenté d'en déduire que l'armature des centres de peuplement présente aujourd'hui bien plus de traits communs avec la situation du milieu du $\mathrm{XIX}^{\mathrm{e}}$ siècle au niveau des villages qu'elle n'en offre à celui des villes moyennes ou grandes.

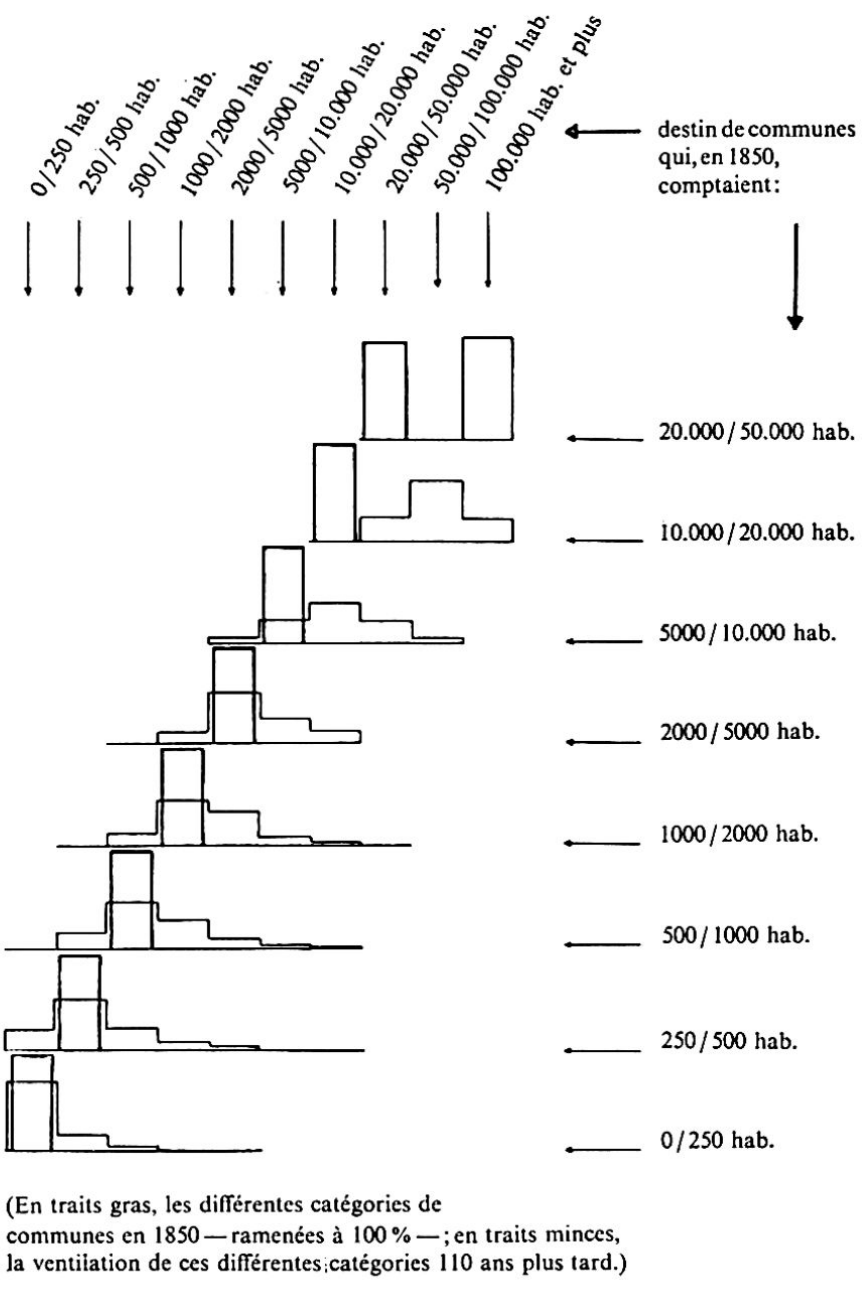


Une analyse précise de ce tableau d'identification conduit cependant à des réflexions plus nuancées. Les agglomérations qui, de nos jours, comptent plus de 10000 habitants, ont toutes augmenté depuis 1850. Et cette promotion s'est effectuée sans modifier, pour l'essentiel, la hiérarchie selon le rang: ou, si l'on veut, ces différentes communes urbaines ont grandi en respectant leur classement de départ de 1850.

Quant aux communes qui groupent actuellement moins de 5000 habitants, elles sont issues d'un triple processus évolutif. Bon nombre d'entre elles, nous l'avons vu, n'ont pas en l'espace de 110 ans changé de catégories, même si à l'intérieur de celles-ci elles ont connu un déclin ou un gain démographique? Une proportion notable provient ensuite des classes de rang moindre. Un contingent plus réduit enfin, se compose de celles qui ont déchu d'une ou - exceptionnellement - de deux classes. Ces niveaux inférieurs présentent donc un mélange de trois types de communes dont deux sont inconnus parmi les catégories urbaines de plus de 10000 âmes: celles qui se sont maintenues et celles qui ont rétrogardé. Si l'on essaie de transposer ces observations statistiques dans une perspective spatiale, on en tire différentes réflexions. Les grandes lignes de la structure urbaine - au sens strict - n'ont fait finalement que s'accuser. Sans doute les rythmes de développement ont-ils été différents selon les villes, entraînant une élongation de la pyramide urbaine. Sans doute aussi le classement a-t-il subi des modifications - détrônant Genève au profit de Zurich par exemple - mais les points d'ancrage majeurs de l'armature se sont affermis, soulignant plus fortement que jamais cette topographie urbaine telle qu'elle s'esquissait il $\mathrm{y}$ a un siècle.

A l'échelle des districts, en revanche, le maillage des centres de dimensions inférieures apparaît comme sensiblement perturbé, puisqu'il juxtapose des communes dont les rapports de tailles se présentaient autrement: il imbrique, en un tissu composite des localités aux tendances démographiques divergentes. Au niveau «rural» par conséquent, les points forts et les points faibles se redistribuent suivant un canevas nouveau. A la différence de ce que l'on observe pour les villes moyennes ou grandes, la carte de 1960 ne peut plus être, ici, la réplique de celle de 1850 .

Et ce sont finalement, les mécanismes même de la différenciation spatiale qui sont concernés. A l'échelle des grands ensembles régionaux, articulés sur les niveaux supérieurs de l'armature urbaine, les inégalités vont procéder, dans le cadre d'une texture peu modifiée, de l'écart numérique grandissant qui sépare les villes: les cités les plus riches en hommes étendant leur ombre sur les autres, à cause probablement et à la mesure en tout cas de leur puissance démographique. A l'échelle des petits espaces,

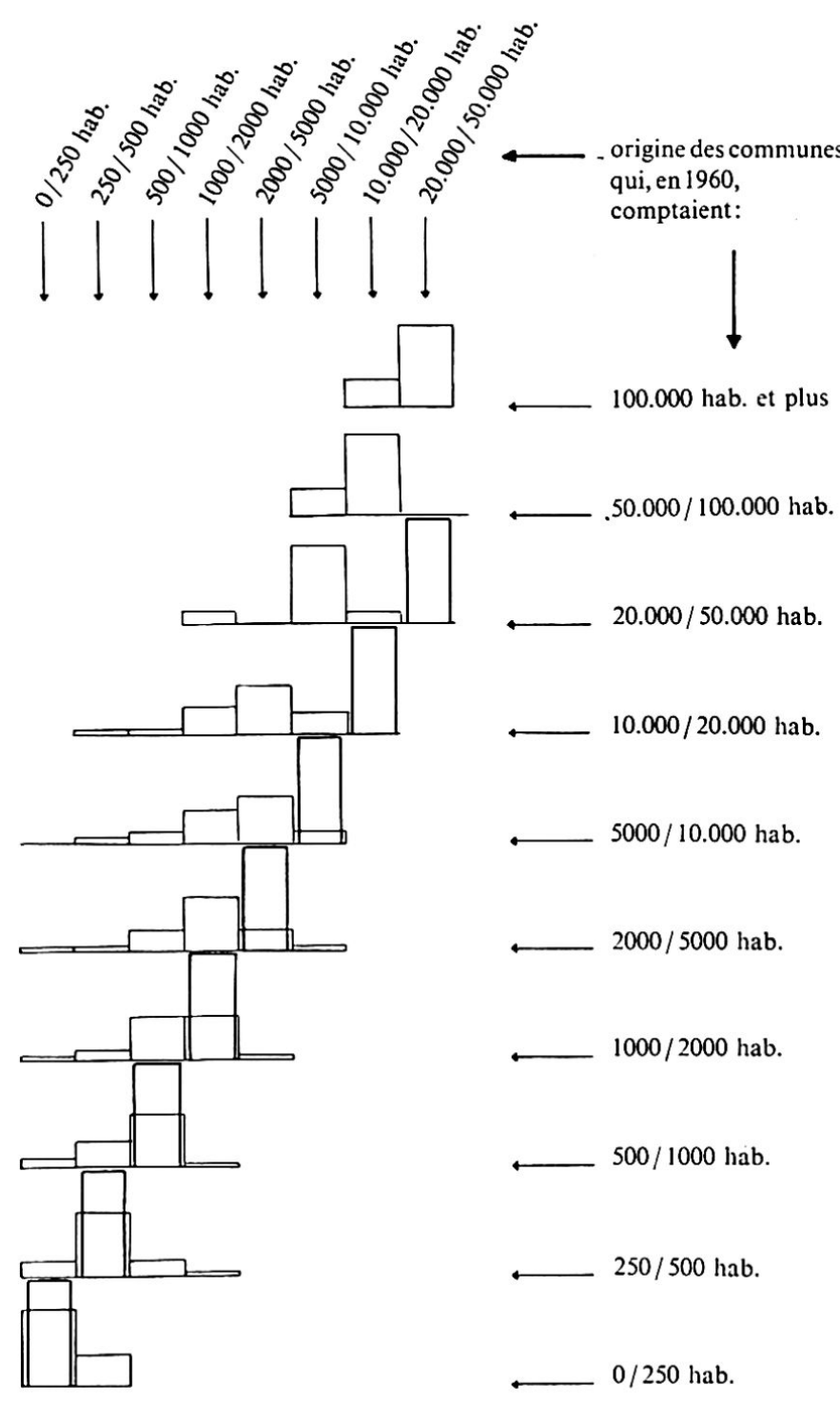

(En traits gras, les différentes catégories de

communes en 1960 - ramenées à $100 \%$-; en traits minces,

la ventilation de ces différentes catégories 110 ans auparavant.)

ces inégalités naîtront de modifications apportées à la texture même du réseau des centres, par la juxtaposition d'essors et de déclins générateurs de relations de voisinage nouvelles. Les disparités macrorégionales et les disparités micro-régionales sont à mettre, chacunes, en corrélation avec la dynamique de l'armature urbaine spécifique à leur niveau: croissance positive inégale pour les premières, combinaisons de croissance, de stabilité et de décroissance pour les secondes.

\section{Remarques}

1 "Quelques aspects de la structure urbaine en Suisse». Cah. de Besançon, No. 16, 1967.

2 Là, évidemment, se pose la question des conventions retenues. Une division plus fine eût fait apparaître sans aucun doute de plus amples changements. Mais, selon toute vraisemblance, les tendances générales resteraient les mêmes. 